\title{
Bi-Stable Spiral Waves: Stepped Spiral Waves
}

\author{
Jian Gao*, Qun Wang, Huaping Lv \\ School of Physics and Electronic Engineering, Jiangsu Normal University, Xuzhou, China \\ Email: *gaojian1612@163.com
}

How to cite this paper: Gao, J., Wang, Q. and Lv, H.P. (2017) Bi-Stable Spiral Waves: Stepped Spiral Waves. Open Access Library Journal, 4: e3982.

https://doi.org/10.4236/oalib.1103982

Received: September 17, 2017

Accepted: November 19, 2017

Published: November 22, 2017

Copyright $\odot 2017$ by authors and Open Access Library Inc.

This work is licensed under the Creative Commons Attribution International License (CC BY 4.0).

http://creativecommons.org/licenses/by/4.0/

\begin{abstract}
A new type of bi-stable spiral waves called "stepped spiral waves", is investigated in this study in an oscillatory medium exhibiting period-doubling bifurcations. Prior to the period-doubling bifurcation of this system, the stepped spiral waves are produced by an unwanted phase trajectory event; the loss of symmetry takes the form of synchronization defect lines, where the trajectory in the local oscillation phase space changes into two different ways. The formation principle of this type of bi-stable spiral wave and the internal structure and geometry of these synchronization defects are studied, and several potential categories of stepped spiral waves are discussed.
\end{abstract}

\section{Subject Areas}

Internal Medicine, Molecular Biology

\section{Keywords}

Renin, Aldosterone, Metabolic Syndrome, Ischaemic Stroke

\section{Introduction}

Spatiotemporal pattern formation in reaction-diffusion (RD) systems is an underlying mechanism responsible for complex biological patterns [1] [2] [3] [4] [5]. Spiral waves are typical patterns in RD systems [6]-[11] which have been observed throughout such diverse systems as chemical reactions [12] [13] [14] [15], fluid convection [16] [17], microorganism populations [18], Xenopus laevis oocytes [19], cardiac muscle [20] [21], chicken retinae [22], and model RD systems [23] [24] [25] [26] [27]. They have been extensively studied both experimentally and theoretically.

A spatially distributed oscillatory medium may undergo bifurcations when the period of the orbit doubles at every point in the system. Prior to the occurrences of these period-doubling bifurcations, the system may support stable rotationally 
symmetric spiral wave solutions in the simple oscillatory regime (period 1) [28]. The dynamics in this regime are typically described by the complex Ginzburg-Landau (CGL) equation, the generic equation for an oscillatory medium near the Hopf bifurcation point at which oscillation appears. Spiral waves are a well-known feature of this regime and have been extensively researched to date [29], however, they can also exist in media with complex periodic or chaotic local dynamics [30] [31] [32]. Goryachev et al. [33], in one such previous investigation of the consequences of period-doubling bifurcation on spiral wave structure and dynamics, identified the wavelength-doubled spiral wave. In this type of wave, the rotational symmetry of the original spiral wave is broken by the presence of synchronization defect lines; the phase of the local orbit changes by multiples of $2 \pi$.

In this paper, we present a new type of spiral wave that is not caused by period-doubling bifurcations in a new complex oscillatory medium. We found that one oscillatory medium can contain two oscillation modes at the same time when there exists a spiral wave. We also determined the mechanism of these oscillation modes, as discussed below.

\section{Model}

We built the model, $\dot{\boldsymbol{c}}(t)=\boldsymbol{G}(\boldsymbol{c}(t))$, to study the movement of a charged particle in a special electric field. Specifically, the calculations described here were carried out on this model, $\boldsymbol{G}(\boldsymbol{c}(t))$ describes the local reaction kinetics, $G_{x}=A c_{y}, G_{y}=B c_{z}, G_{z}=C c_{z}+q_{1} c_{y}+q_{2} c_{x}+q_{3} c_{x}^{3}$. And $c_{x}$ is the location of the particle, $c_{y}$ is its speed, and $c_{z}$ is the acceleration of it. The rate of change of the special electric field is a function of $c_{x}, c_{y}$ and $c_{z}$. In this electric field, chaotic motion of the movement of charged particle can occur. We have considered cases where the spatially homogeneous system $\dot{\boldsymbol{c}}(t)=\boldsymbol{G}(\boldsymbol{c}(t))$ exhibits period-doubling bifurcation. As shown in the figure, when parameter $C \approx-0.40$ $\left(A=1.00 \mathrm{~s}, B=1.00 \mathrm{~s}, q_{1}=-1.00 \mathrm{~s}^{-1}, q_{2}=-1.00 \mathrm{~s}^{-2}, q_{3}=1.00 \mathrm{~s}^{-6}\right)$, period doubling bifurcation occurs. Numerical simulations show that the system enters chaos state via a period-doubling bifurcation route. But this period-doubling bifurcation doesn't play a central role in the organization of stepped spiral wave which will be discussed below. Figure 1(a) shows the bifurcation diagram of the equation. The parameter $C$ is set to a control parameter, and recorded the maximum of $c_{x}$. As shown in Figure 1(a), the bifurcation graph is not continuous when period-3 occurs, which is a strange phenomenon. The reason of this strange phenomenon is phase trajectory catastrophe (the phase point turns from one limit cycle into the other one unpredictably). And this plays a central role in the organization of super-spiral structure. As shown in Figure 1(b), this system has three fixed points in the parameter region we provided above: $O(0,0,0), P^{+}$ $(+1,0,0)$ and $P^{-}(-1,0,0) . \mathrm{O}$ is an unstable focus-node point which is a stable node in one direction and an unstable focus in two directions. $P^{+}$and $P^{-}$are unstable focus-node points that are unstable node in one direction and stable focuses in two directions. Specifically, this system has two different limit cycles in 

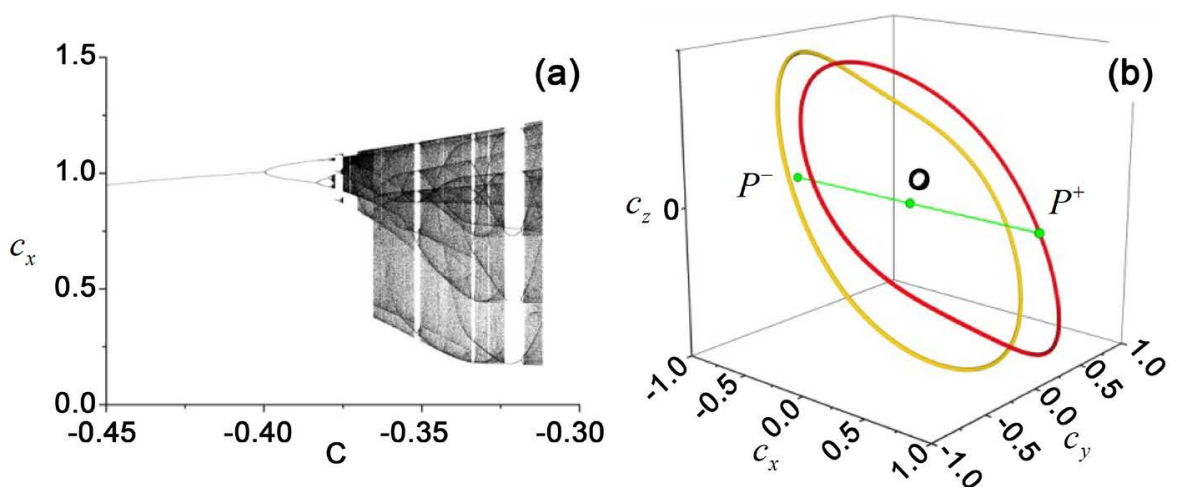

(c)

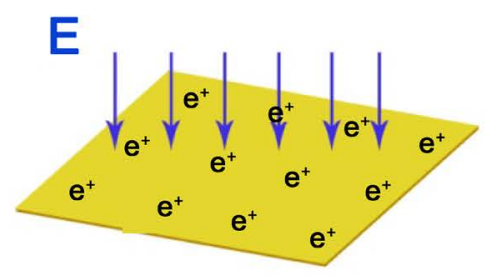

Figure 1. (Color online) (a) Bifurcation diagram of proposed equation. Parameter $C$ is set to a control parameter with recorded maximum of $c_{x}$; (b) Phase trajectory structures of the spatially homogeneous system in Equation (1). Parameters are $A=1.00 \mathrm{~s}$, $B=1.00 \mathrm{~s}, C=-0.42, q_{1}=-1.00 \mathrm{~s}^{-1}, q_{2}=-1.00 \mathrm{~s}^{-2}, q_{3}=1.00 \mathrm{~s}^{-6}$.

the region which plays a central role in the organization of the super-structure spiral waves $(C=-0.42)$. One limit cycles is symmetrical with the other one about the fixed point $\mathrm{O}$ as shown in Figure 1(a). Under some conditions, such as parameter $C$ changes, phase trajectory catastrophe will occur.

Equation $\dot{\boldsymbol{c}}(t)=\boldsymbol{G}(\boldsymbol{c}(t))$ describes the movement (one-dimensional motion) of the charged particles in a rapidly alternating electric field. Then the movement of the uniformly charged oil slick (see Figure 1(c)) can be described by the equation as follows:

$$
\begin{gathered}
\frac{\partial \boldsymbol{c}(\boldsymbol{r}, t)}{\partial t}=\boldsymbol{G}(\boldsymbol{c}(\boldsymbol{r}, t))+\boldsymbol{D} \nabla^{2} \boldsymbol{c}(\boldsymbol{r}, t) \\
\boldsymbol{D}=\left[\begin{array}{ccc}
D_{x} & 0 & 0 \\
0 & D_{y} & 0 \\
0 & 0 & D_{z}
\end{array}\right]
\end{gathered}
$$

where $\boldsymbol{c}(\boldsymbol{r}, t)$ is a vector, $\boldsymbol{D}$ is the diffusion coefficient. The movement of one point can drive the points around it, this effect is similar to diffusing phenomenon. Various values of the parameter $C$ in the interval $[-1.0,-0.3]$ were considered, while the other parameters were fixed at $A=1.00 \mathrm{~s}$ and $B=1.00 \mathrm{~s}$. The scaled diffusion coefficient was $D_{x, z} \Delta t /(\Delta r)^{2}=1.5 \times 10^{-2}, D_{y} \Delta t /(\Delta r)^{2}=0$ $\left(\Delta t=2.0 \times 10^{-2} \mathrm{~s}\right)$ in all calculations.

We conducted simulations in a disk-shaped domain of radius 600 with no-flux boundary conditions. Equation (1) was integrated with an explicit fourth-order Runge-Kutta method, where the Laplace operator was approximated with nine nearest-neighbor sites. When the explicit fifth-order Runge-Kutta algorithm was used, there was no qualitative difference in the observed spatiotemporal behavior. Further, the same results were obtained when smaller space steps and time steps were used, indicating that the phenomena reported here cannot be ascribed to any improper selection of integration methods, step size, or other such factors.

\section{Results and Discussion}

Figure 2 shows the $c_{x}\left(\boldsymbol{r}, t_{0}\right)$ concentration field at a single time instant $t_{0}$. In 


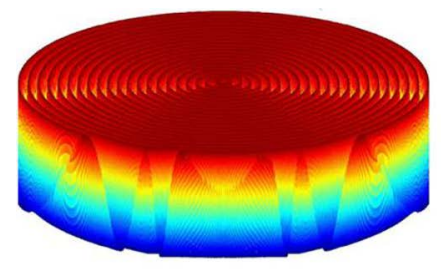

(a)

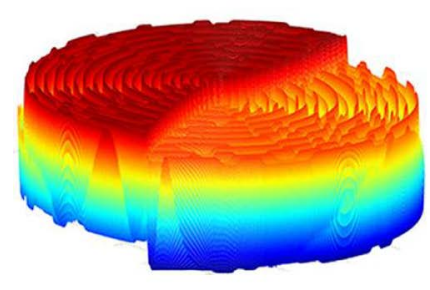

(c)

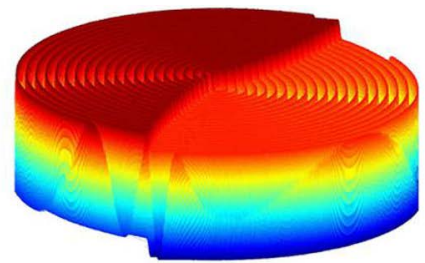

(b)

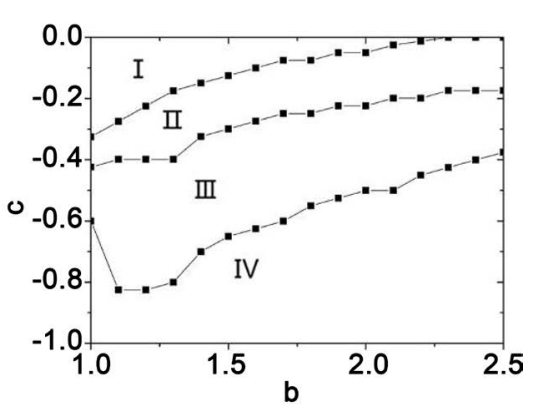

(d)

Figure 2. (Color online) In the case of $A=1.00 \mathrm{~s}, B=1.00 \mathrm{~s}, C=-0.42, q_{1}=-1.00 \mathrm{~s}^{-1}$, $q_{2}=-1.00 \mathrm{~s}^{-2}, q_{3}=1.00 \mathrm{~s}^{-6}$, in structures of spiral waves for different $C$, each pattern is based on the same initial spatial values. $C$ is (a): -0.50 ; (b): -0.40 ; (c): -0.33 ; (d): Phase diagram revealing regions of different wave forms; I and IV: Medium cannot support spiral waves; II: Medium can support stepped spiral waves; III: Medium can support simple spiral waves. Simulations were carried out on a disk-shaped domain of radius 600.

cases where $C \leq-0.43$ was part of the period-1 regime, the medium supported a single, stable, one-armed spiral wave (Figure 2(a)). As the parameter $C$ increased, the spiral wave acquired a global structure different from that in a simple periodic medium prior to the period-doubling bifurcation of local dynamics $(-0.43 \leq C \leq-0.35$ ) (Figure $2(b)$ ). In the case of $C \approx-0.35$ (i.e., a spatially homogenous model with bifurcation at $C^{*} \approx-0.40$.), the local dynamics underwent a period-doubling bifurcation and the spiral wave structure changed again (Figure 2(c). Figure 2(d) shows the $B$ - $C$ parameter space revealing regions of different wave forms.

The whole spiral wave is divided into two parts by a curve (Figure 3(a)). Although the spiral rotates, the curve is stationary up to numerical accuracy. The lower panel of Figure 3(a) shows $c_{x}\left(\boldsymbol{r}, t_{0}\right)$ in various colors indicating the curve connecting the spiral core and boundaries, denoted as $\Omega$, where sharp changes in concentration occur. This spiral wave is different, in appearance and mechanism, from the wavelength-doubled spiral wave (Figure 3(b)) described by Goryachev et al. in 1998 as being caused by period-doubling bifurcations [33].

In the asymptotic regime, after the spiral reaches its steady state, the shape of the $\Omega$ curve is a straight line segment with a short curved portion lying inside the core region. In the case of $-0.43 \leq C \leq-0.35$, the phase trajectory of the point on one side of the $\Omega$ curve, a stable limit cycle (period-1), is different from that on the other side. In other words, the points on either side of the curve 


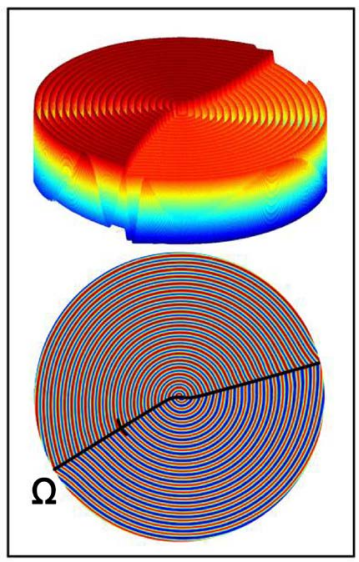

(a)

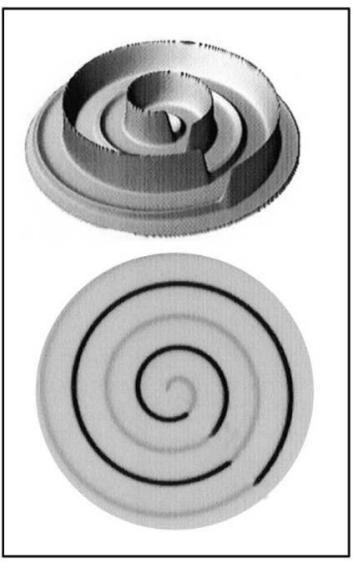

(b)

Figure 3. (Color online) Comparison among both categories of spiral waves. (a): In the case of $C=-0.40$, the other parameters are the same as in Figure 2; (a): Concentration field $c_{x}\left(r, t_{0}\right)$ is shown as elevation in the upper panel and as grey shades in the lower Panel; solid line depicts the $\Omega$ curve; (b): Wavelength-doubled spiral wave identified by Goryachev et al. [33]. Also shown: Arc segment at radius $r_{0}=300$ along which points were taken to construct Figure 4.

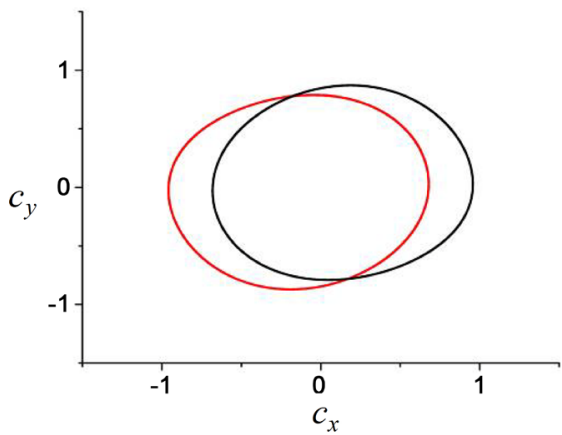

(a)

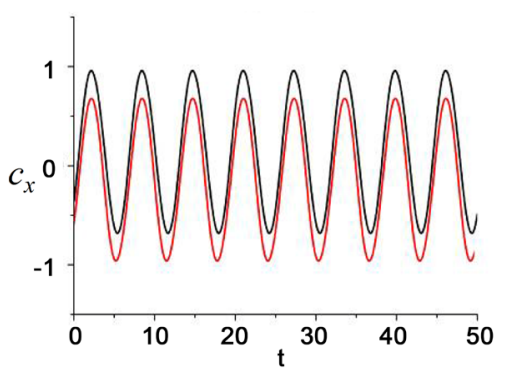

(c)

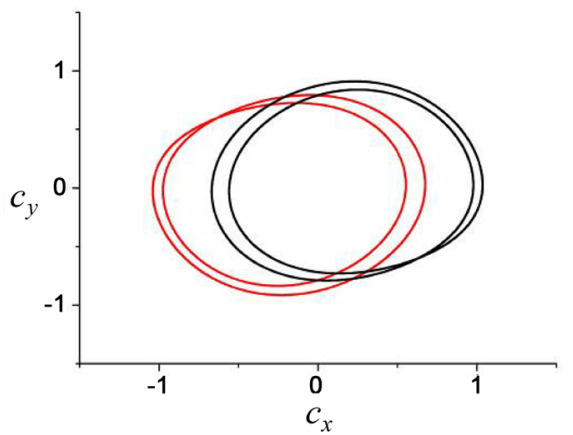

(b)

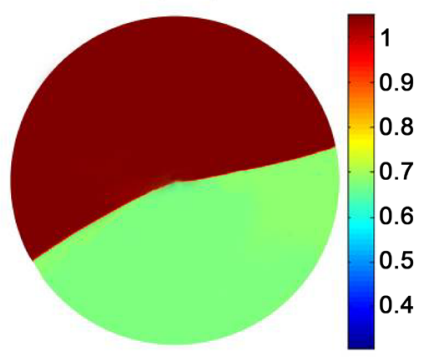

(d)

Figure 4. (Color online) (a), (b): Loop exchange in local orbits as the $\Omega$ curve along the arc at radius $r_{0}=300$ indicated in the lower panel of Figure 3(a). $\theta_{1}=\theta_{\Omega}-0.05$ (black line); $\theta_{2}=\theta_{\Omega}+0.05$ (red line); (c) Two $c\left(r, t_{0}\right)$ concentration time series calculated for the medium at the two points; (d) Maximum concentration $c\left(r, t_{0}\right)$ distribution in the medium supporting spiral waves.

have different stable oscillation states (Figure 4(a)). Within the period-2 domain, the structure of the spiral wave on each side of the $\Omega$ curve takes the form of half of the wavelength-doubled spiral wave; on one side of the $\Omega$ curve, high 
and low wave crests are alternately arranged. The points on both sides of the curve are then also in different stable oscillation states (period-2) as shown in Figure 4(b).

Figure 4(c) shows two $c_{x}(r, t)$ time series (period-1) of the two spatial points on either side of the curve (across the $\Omega$ curve along the arc): The phases of oscillation are the same, but at the maximum and minimum. We observed that the maximum (minimum) concentrations of every point on each side of the $\Omega$ curve are the same, and the maximum (minimum) concentration on the left of the curve is always greater (lower) than the other side of the curve (Figure 4(d)). This phenomenon is crucial to the organization of the spiral wave; the corresponding principle is discussed in detail below.

Consider again the spatially homogeneous system $\dot{\boldsymbol{c}}(t)=\boldsymbol{G}(\boldsymbol{c}(t))$. A new variable $\boldsymbol{C}(t)=\boldsymbol{c}(t)-c_{0} \quad\left(c_{0}\right.$ is the fixed point $)$ can be defined so that the solution near the fixed point is $C(t)=J_{1} \mathrm{e}^{\lambda_{1} t}+J_{2} \mathrm{e}^{\lambda_{2} t}+J_{3} \mathrm{e}^{\lambda_{3} t}$. Near fixed point $O$, there are the following function relationships (approximate solutions):

$$
\begin{aligned}
& \lambda_{O 1} \approx k_{O 1}(C+1)-1.05 \\
& \lambda_{O 2, O 3} \approx k_{O 2}(C+1)+0.02 \pm i \beta_{O 0} \\
& k_{O 1}, k_{O 2} \geq 0
\end{aligned}
$$

near $P^{\dagger}$ and $P^{-}$:

$$
\begin{aligned}
& \lambda_{P 1} \approx k_{P 1}(C+1)-0.85 \\
& \lambda_{P 2, P 3} \approx k_{P 2}(C+1)-+0.75 \pm i \beta_{P 0} \\
& k_{P 1}, k_{P 2} \geq 0
\end{aligned}
$$

where $\beta$ can be any real number, $k$ is the slope of the line, and $\lambda$ is the function of $C$.

According to Equations ((2) and (3)), the attraction regions of $P^{\dagger}$ or $P^{-}$decrease as parameter $C$ increases while the affected area of $O$ increases as $C$ increases. There could exist a single stable limit cycle in phase space when the overlap-area of $P^{\dagger}$ and $P^{-}$attraction regions exceeds the critical level: the attraction regions of $P^{\dagger}$ and $P^{-}$gradually decrease as $C$ increases, and the phase orbit tends to rotate around $P^{\dagger}$ or $P^{-}$.

Figure 5 shows the attractor of this model on $\left(c_{x}, c_{y}\right)$ plane with increase in parameter $C$. At $C \approx-0.35$, the phase orbit rotates around two different points $P^{\dagger}$ and $P^{-}$. In some regions near fixed point $P^{\dagger}$ of the phase space, the orbit tends to rotate around $P^{\dagger}$; the same is true for $P^{-}$. As shown in Figure 6, this attractor is significantly different from the Rössler attractor: The former has two unstable equilibrium points while the latter has only one. This is why the phase orbit tends to rotate around $P^{\dagger}$ or $P^{-}$under the same conditions. The phase orbit can only rotate around one single point in the Rössler model, however, regardless of the parameters. So, in a Rössler medium, changes in spiral wave structure are due solely to period-doubling bifurcation. This phenomenon is responsible for the difference between stepped spiral waves and wavelength-doubled spiral waves.

When the parameter $C$ exceeds the critical value, the medium splits into two 


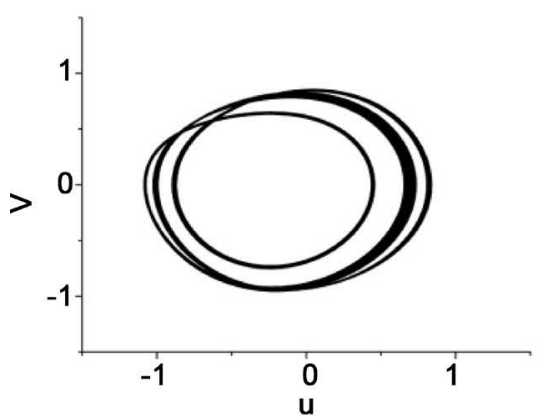

(a)

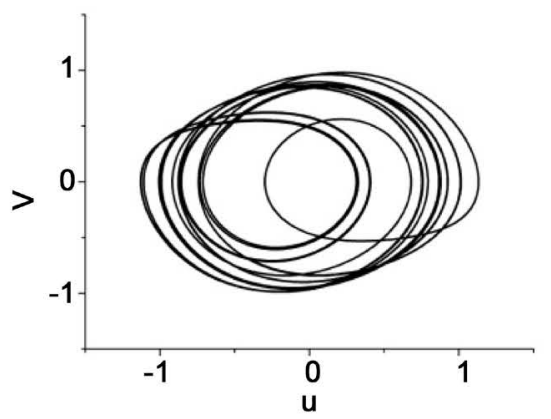

(b)

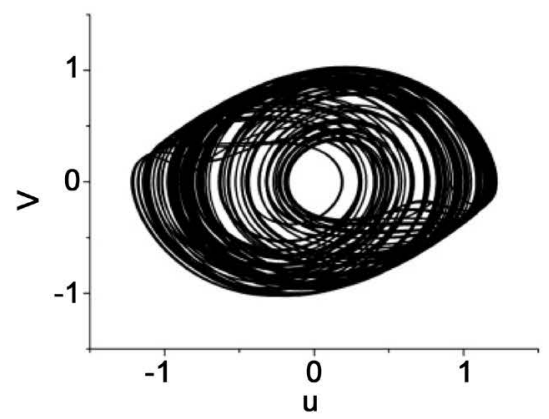

(c)

Figure 5. (Color online) Structures of phase trajectories for varying parameter $C$, other parameters are the same as Figure 2 . $C$ is (a): -0.375 ; (b): $-0.350 ;(\mathrm{c}):-0.313$.

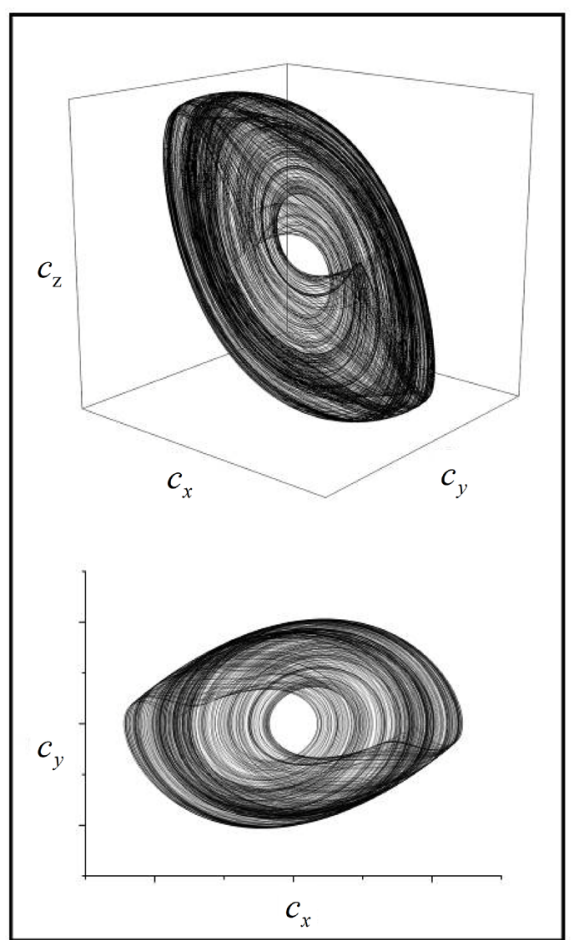

(a)

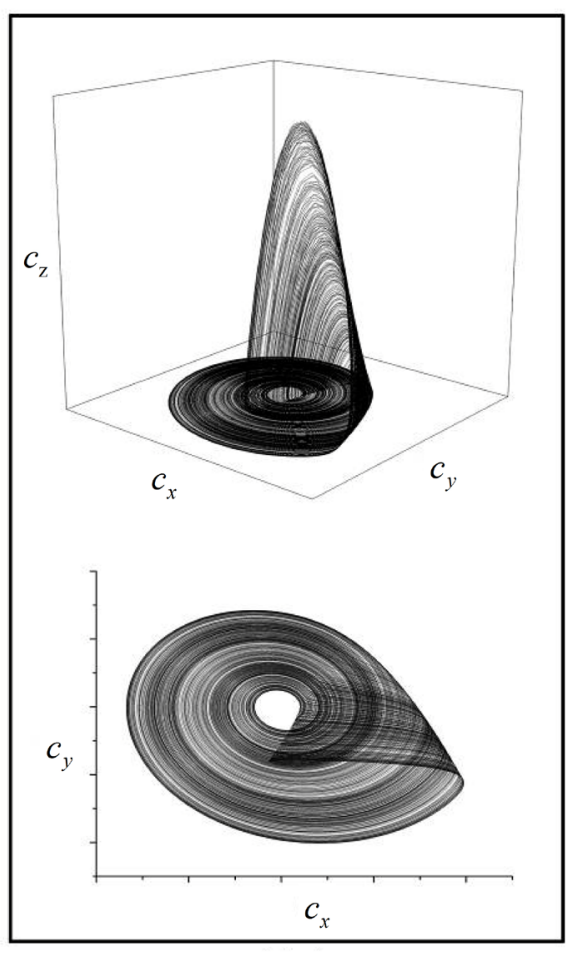

(b)

Figure 6. (Color online) Comparison of proposed attractor (a) and Rössler attractor (b) structures.

kinds of oscillation forms based on the initial conditions (i.e., unrelated to perioddoubling bifurcation). As shown in Figure 7, the spiral tip trajectory is a perfect circle containing half high concentration and half low concentration. The boundaries of high and low concentration form a straight line passing through the center of the circle. When the spiral tip enters $S_{1}$, it forms the oscillation of $S_{1}$; when it enters $\mathrm{S}_{2}$, it forms $S_{2}$. The duration that the spiral tip stayed in $S_{1}$ or $S_{1}$ is half of the spiral wave period. The spiral tip produces two categories of waves: High (high concentration) and low (low concentration) waves, which spread toward the boundary of the medium. The spiral tip is organization center of the entire spiral wave in a given reaction-diffusion system [34]. These two categories 


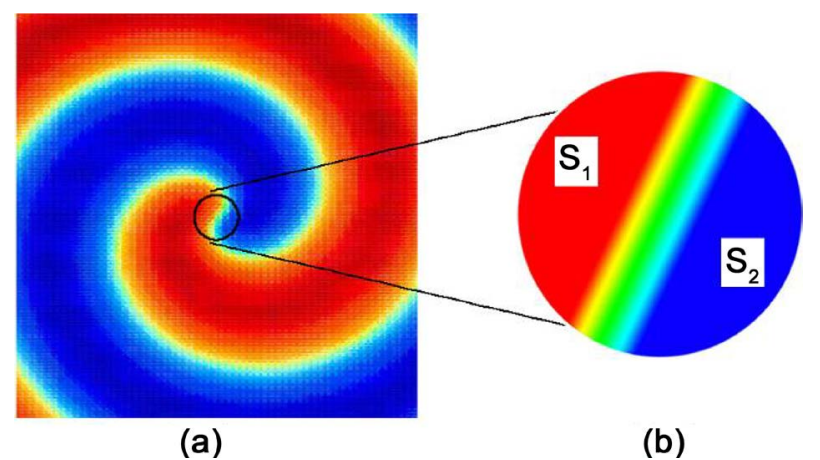

Figure 7. (Color online) (a) Black circle marks spiral tip trajectory; (b) Partial enlargement inside (a).

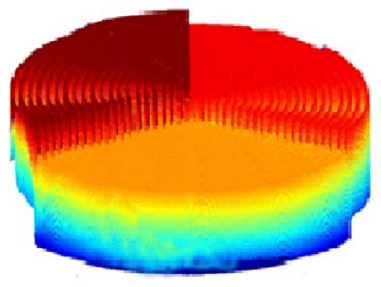

(a)

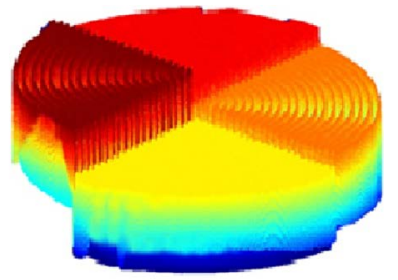

(b)

Figure 8. (Color online) Structures of possible spiral waves in a system with multi-scroll attractors. (a) Potential spiral wave in a system with three-scroll attractors; (b) Potential spiral wave in a system with four-scroll attractors.

of waves are eventually evenly divided throughout the whole medium.

\section{Summary}

In this study, we discovered a novel complex-structure spiral wave called the stepped spiral wave, which formed in bi-stable media. According to the initial conditions, a phase trajectory catastrophe may occur with changes in parameter $C$ causing the spiral tip to produce two types of wave: High (high concentration) and low (low concentration) waves, which spread towards the boundary of the medium. We expect this type of super-spiral structure can be observed in laboratory. Our numerical results suggest that these stepped spiral structures can be observed experimentally; we conjecture that a system with multi-scroll attractors may support stepped spiral waves. When the number of scrolls is three, the system may support a stepped spiral wave as shown in Figure 8(a). When the number is four, the system may support a spiral wave as shown in Figure 8(b). In the future, we plan to conduct further studies to support this conjecture.

\section{Acknowledgements}

This project was supported by the Natural Science Foundation of China (under grant no. 10335010) and the research of pattern dynamics of complex space-time system and control (no. 11175150). J. Gao. was supported by the Priority Academic Program Development of Jiangsu Higher Education Institutions (PAPD) and the Research Fund of Jiangsu Normal University under grant no. 12XLR028. 


\section{References}

[1] Turing, A.M. (1952) Philosophical Transactions of the Royal Society of London. Series B, Biological Sciences, 237, 37. https://doi.org/10.1098/rstb.1952.0012

[2] Schnell, S., Grima, R. and Maini, P.K. (2007) Multiscale Modeling in Biology. American Scientist, 95, 134. https://doi.org/10.1511/2007.64.134

[3] Meinhardt, H. (2003) The Algorithmic Beauty of Sea Shells. Springer, Berlin. https://doi.org/10.1007/978-3-662-05291-4

[4] Murray, J.D. (2003) Mathematical Biology. Springer, New York.

[5] Woolley, T.E., Baker, R.E., Maini, P.K., et al. (2010) Analysis of Stationary Droplets in a Generic Turing Reaction-Diffusion System. Physical Review E, 82, Article ID: 051929. https://doi.org/10.1103/PhysRevE.82.051929

[6] Cross, M.C. and Hohenberg, P.C. (1993) Pattern Formation Outside of Equilibrium. Reviews of Modern Physics, 65, 851. https://doi.org/10.1103/RevModPhys.65.851

[7] Aranson, I.S. and Kramer, L. (2002) The World of the complex Ginzburg-Landau Equation. Reviews of Modern Physics, 74, 99. https://doi.org/10.1103/RevModPhys.74.99

[8] Kuramoto, Y. (1984) Chemical Oscillations, Waves, and Turbulence. Springer-Verlag, Berlin. https://doi.org/10.1007/978-3-642-69689-3

[9] Mikhailov, A.S. and Showalter, K. (2006) Control of Waves, Patterns and Turbulence in Chemical Systems. Physics Reports, 425, 79. https://doi.org/10.1016/j.physrep.2005.11.003

[10] Yang, X., Marenduzzo, D. and Marchetti, M.C. (2014) Spiral and Never-Settling Patterns in Active Systems. Physical Review E, 89, Article ID: 012711. https://doi.org/10.1103/PhysRevE.89.012711

[11] Affan, H. and Friedrich, R. (2014) Spiral Defect Chaos in an Advection-Reaction-Diffusion System. Physical Review E, 89, Article ID: 062920. https://doi.org/10.1103/PhysRevE.89.062920

[12] Winfree, A.T. (1972) The Revival Wave Induced Spontaneously in a BZ-Type Mixture. Science, 175, 634. https://doi.org/10.1126/science.175.4022.634

[13] Ouyang, Q., Swinney, H.L. and Li, G. (2000) Transition from Spirals to Defect-Mediated Turbulence Driven by a Doppler Instability. Physical Review Letters, 84, 1047. https://doi.org/10.1103/PhysRevLett.84.1047

[14] Vanag, V.K. and Epstein, I.R. (2001) Inwardly Rotating Spiral Waves in a Reaction-Diffusion System. Science, 294, 835. https://doi.org/10.1126/science.1064167

[15] Zanin, M.P., Hellström, M., Shepherd, R.K., Harvey, A.R. and Gillespie, L.N. (2014) Development of a Cell-Based Treatment for Long-Term Neurotrophin Expression and Spiral Ganglion Neuron Survival. Neuroscience, 277, 26.

[16] Pinter, A., Lücke, M. and Hoffmann, Ch. (2006) Wave-Number Dependence of the Transitions between Traveling and Standing Vortex Waves and Their Mixed States in the Taylor-Couette System. Physical Review Letters, 96, Article ID: 044506. https://doi.org/10.1103/PhysRevLett.96.044506

[17] Gu, C., St-Yves, G. and Davidsen, J. (2013) Spiral Wave Chimeras in Complex Oscillatory and Chaotic Systems. Physical Review Letters, 111, Article ID: 134101. https://doi.org/10.1103/PhysRevLett.111.134101

[18] Tomchik, K.N. and Devreotes, P.N. (1981) Adenosine 3',5'-Monophosphate Waves in Dictyostelium Discoideum: A Demonstration by Isotope Dilution-Fluorography. Science, 212, 433. https://doi.org/10.1126/science.6259734 
[19] Lechleiter, J., Girard, S., Peralta, E. and Clapham, D. (1991) Spiral Calcium Wave Propagation and Annihilation in Xenopus laevis Oocytes. Science, 252, 123. https://doi.org/10.1126/science.2011747

[20] Hörning, M., Takagi, S. and Yoshikawa, K. (2010) Wave Emission on Interacting Heterogeneities in Cardiac Tissue. Physical Review E, 82, Article ID: 021926. https://doi.org/10.1103/PhysRevE.82.021926

[21] Tang, J., Luo, J., Ma, J., Yi, M. and Yang (2013) Spiral Waves in Systems with Fractal Heterogeneity. Physica A: Statistical Mechanics and Its Applications, 392, 22.

[22] Dahlem, M.A. and Müller, S.C. (1997) Control of Sub-Excitable Waves in Neural Networks by Nonlocal Coupling. Experimental Brain Research, 115, 319. https://doi.org/10.1007/PL00005700

[23] Zhan, M. and Kapral, R. (2006) Destruction of Spiral Waves in Chaotic Media. Physical Review E, 73, Article ID: 026224. https://doi.org/10.1103/PhysRevE.73.026224

[24] Sridhar, S., Sinha, S. and Panfilov, A.V. (2010) Anomalous Drift of Spiral Waves in Heterogeneous Excitable Media. Physical Review E, 82, Article ID: 051908. https://doi.org/10.1103/PhysRevE.82.051908

[25] Sandstede, B. and Scheel, A. (2001) Superspiral Structures of Meandering and Drifting Spiral Waves. Physical Review Letters, 86, 171. https://doi.org/10.1103/PhysRevLett.86.171

[26] Bär, M. and Brusch, L. (2004) Breakup of Spiral Waves Caused by Radial Dynamics: Eckhaus and Finite Wavenumber Iinstabilities. New Journal of Physics, 6, 5. https://doi.org/10.1088/1367-2630/6/1/005

[27] Luengviriya, J., Sutthiopad, M., Phantu, M., Porjai, P., Kanchanawarin, J., Müller, S.C. and Luengviriya, C. (2014) Meandering Spiral Waves in a Bubble-Free Belousov-Zhabotinsky Reaction with Pyrogallol. Physical Review E, 90, Article ID: 052919. https://doi.org/10.1103/PhysRevE.90.052919

[28] Petrov, V., Ouyang, Q. and Swinney, H.L. (1997) Resonant Pattern Formation in a Chemical System. Nature (London), 388, 655. https://doi.org/10.1038/41732

[29] Aranson, L.K. and Weber, A. (1993) Theory of Interaction and Bound States of Spiral Waves in Oscillatory Media. Physical Review E, 47, 3231. https://doi.org/10.1103/PhysRevE.47.3231

[30] Klevecz, R., Pilliod, J. and Bolen, J. (1991) Autogenous Formation of Spiral Waves by Coupled Chaotic Attractors. Chronobiology International, 8, 6. https://doi.org/10.3109/07420529109063914

[31] Brunnet, L., Chaté, H. and Manneville, P. (1994) Long-Range Order with Local Chaos in Lattices of Diffusively Coupled ODEs. Physica (Amsterdam), 78D, 141.

[32] Goryachev, A. and Kapral, R. (1996) Structure of Complex-Periodic and Chaotic Media with Spiral Waves. Physical Review E, 54, 5469. https://doi.org/10.1103/PhysRevE.54.5469

[33] Goryachev, A. and Chaté, H. (1998) Transitions to Line-Defect Turbulence in Complex Oscillatory Media. Physical Review Letters, 80, 4.

[34] Başar, G. and Dunne, G.V. (2010) Chiral Magnetic Spirals. Physical Review Letters, 104, 23. https://doi.org/10.1103/PhysRevLett.104.232301 